\title{
(6) OPEN ACCESS \\ Common mental disorders and mortality in the West of Scotland Twenty-07 Study: comparing the General Health Questionnaire and the Hospital Anxiety and Depression Scale
}

\author{
Mary Kathleen Hannah, ${ }^{1} \mathrm{G}$ David Batty, ${ }^{2,3}$ Michaela Benzeval ${ }^{1}$
}

${ }^{1} \mathrm{MRC} / \mathrm{CSO}$ Social and Public Health Sciences Unit, Glasgow, UK

${ }^{2}$ Department of Epidemiology \& Public Health, University College London, London, UK ${ }^{3} \mathrm{MRC}$ Centre for Cognitive Ageing and Cognitive Epidemiology, Department of Psychology, University of Edinburgh, Edinburgh, UK

Correspondence to Mary Kathleen Hannah, MRC/CSO Social and Public Health Sciences Unit, 4 Lilybank Gardens, Glasgow G12 8RZ, UK; m.hannah@sphsu.mrc.ac.uk

Received 11 September 2012 Revised 15 February 2013 Accepted 9 March 2013 Published Online First 29 March 2013

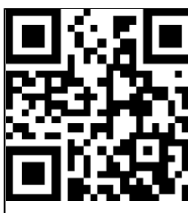

Open Access Scan to access $m$ free antent

To cite: Hannah MK, Batty GD, Benzeval M. J Epidemiol Community Health 2013;67:558-563.

\section{ABSTRACT}

Background While various measures of common mental disorders (CMD) have been found to be associated with mortality, a comparison of how different measures predict mortality may improve our understanding of the association. This paper compares how the Hospital Anxiety and Depression Scale (HADS) and the 30-item General Health Questionnaire (GHQ-30) predict all cause and cause-specific mortality. Methods Data on 2547 men and women from two cohorts, aged approximately 39 and 55 years, from the West of Scotland Twenty-07 Study who were followed up for mortality over an average of 18.9 (SD 5.0) years. Scores were calculated for HADS depression (HADS-D), HADS Anxiety (HADS-A) and GHQ-30. Cox Proportional Hazards Models were used to determine how each CMD measure predicted mortality.

Results After adjusting for serious physical illness, smoking, social class, alcohol, obesity, pulse rate and living alone, HRs $(95 \% \mathrm{CI})$ per SD increase in score for all-cause mortality were: 1.15 (1.07 to 1.25) for HADSD; 1.13 (1.04 to 1.23) for GHQ-30 and 1.05 (0.96 to 1.14) for HADS-A. After the same adjustments, cardiovascular disease mortality was also related to HADS-D (HR 1.24 (1.07 to 1.43)), to GHQ-30 (HR 1.24 (1.11 to 1.40$)$ ) and to HADS-A (HR 1.15 (1.01 to 1.32)); respiratory mortality to GHQ-30 (HR 1.33 (1.13 to 1.55$))$ and mortality from other causes, excluding injuries, to HADS-D (HR 1.28 (1.05 to 1.55)).

Conclusions There were associations between CMD and both all-cause and cause-specific mortality which were broadly similar for GHQ-30 and HADS-D and were still present after adjustment for important confounders and mediators.

\section{INTRODUCTION}

The Hospital Anxiety and Depression Scale (HADS) ${ }^{1}$ and the 30 -item General Health Questionnaire (GHQ-30) ${ }^{2}$ are two self-completion inventories used in population surveys as measures of common mental disorders (CMD). While both have been shown to predict mortality ${ }^{3-8}$ each has different qualities that could influence the association: while the 30-item GHQ was designed to screen for general psychiatric disorders, including anxiety and depression, HADS consists of two seven-item subscales, each designed specifically to discriminate between anxiety and depression. Anxiety and depression have shown different associations with mortality, ${ }^{59}$ but it is not known how a general measure of CMD such as GHQ-30 would compare. There may be other differences between the two instruments that could affect their association with mortality: although both ask about the recent past, unlike HADS, the 'as usual' wording of GHQ may limit its ability to capture chronic CMD; even though each has been designed to be unaffected by physical symptoms, ${ }^{12}$ GHQ in particular has been criticised as containing items relating to physical symptoms. ${ }^{10}$ Any differences or similarities observed may help shed light on the mechanisms linking CMD to mortality.

In order to investigate any differences or similarities between the instruments, we conducted a systematic and simultaneous comparison of the relationship of GHQ-30 and HADS with mortality using data from the West of Scotland Twenty-07 Study.

\section{METHODS}

\section{Participants}

The Twenty-07 Study ${ }^{11}$ followed men and women in three age cohorts-born around 1932, 1952 and 1972_for 20 years. It has two subsamples: the regional sample, a two-stage stratified random sample of people living in the Central Clydeside Conurbation, West of Scotland and the localities sample of people from two areas of the city of Glasgow. Of those who agreed to have their contact details passed from the local authority to the Medical Research Council, 87\% took part in Wave 1 interviews conducted in 1987/1988; the achieved sample was 4510 (target 1500 per cohort). The second wave was conducted in 1990-1992, with $86 \%$ of those issued taking part. Ethical approval for Wave 1 was granted by the ethics subcommittee of the West of Scotland Area Medical Committees and the GP Sub-Committee of Greater Glasgow Health Board; the latter also approved Wave 2. Written consent was given for each wave. Cohort members are flagged with the Scottish National Health Service Central Registry for mortality follow-up: which provides International Classification of Diseases (ICD) codes for cause of death. Wave 1 respondents have been shown to be representative of the general population of the sampled area. ${ }^{12}$

The data used here are from the two older cohorts and baseline is the first wave in which data on both GHQ-30 and HADS were available: for the 1930s cohort it is Wave 1 , when they were aged around 55 years, and for the 1950s cohort it is Wave 2, when they were aged around 39 years. Figure 1 illustrates the numbers included from each of these cohorts. 


\section{Mortality}

As of June 2011, participants had been followed up for a maximum of 23.2 years (mean 18.9 years, SD 5.0). The mortality outcomes used were: all-cause mortality; cardiovascular diseases (CVD): (ICD) 9 codes 390-459.9; ICD 10 codes: I00I99; respiratory diseases: ICD 9 codes 460-519.9; ICD 10 codes: J00-J99.8; all cancers: ICD 9 codes 140-239.9; ICD 10 codes: C00-D48.9 and injuries: ICD 9 codes 800-999; E800E999; ICD 10 codes: S00-T983; V01-Y98.

\section{Measures of common mental disorder}

The HADS ${ }^{1}$ was designed for use in a non-psychiatric hospital setting but has been used in other clinical and general population settings, and correlated well with interview-based measures and other screening questionnaires that identify psychiatric distress. ${ }^{13}$ It contains 14 items selected to be relatively unaffected by physical illness ${ }^{1}$ and has two subscales: anxiety and depression, each with seven items scored on a four-point scale between zero and three, creating a maximum score of 21 on each subscale. If only one item on a subscale was missing, its value was inferred as the mean of the available six items. ${ }^{14}$ Continuous scores were used for all analyses. HADS anxiety covers anxious mood, restlessness and anxious thoughts but not the somatic symptoms of anxiety; HADS depression focuses on loss of interest and diminished pleasure response. ${ }^{1}$

The GHQ-30, ${ }^{2}$ a self-administered inventory designed to be used in the general population and within non-psychiatric clinical settings, comprises 30 questions, covering a range of neurotic symptoms with an emphasis on those typical of anxiety and depression with a deliberate avoidance of those that might also reflect physical illness. The responses are made on a four-point scale between zero and three. For all analyses, continuous scores

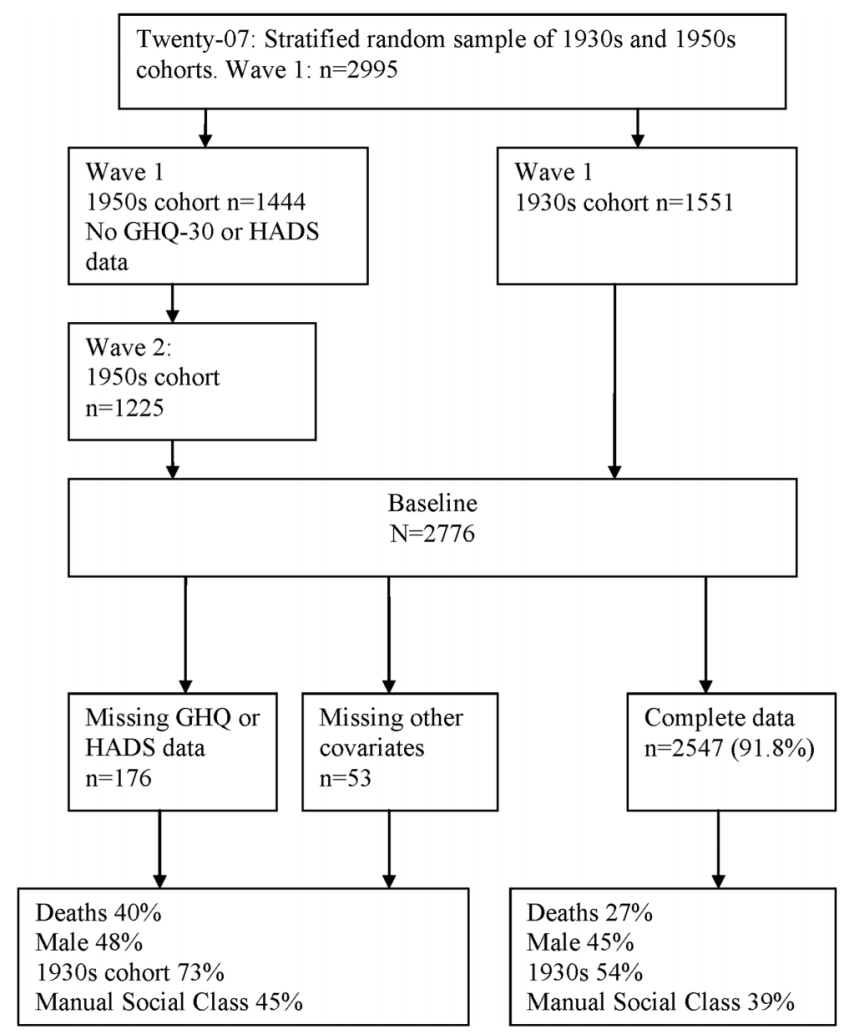

Figure 1 Flow of Study Members through the West of Scotland Twenty-07 Study, 1930s and 1950s cohort. were used and these were calculated by summing (potential range 0-90). Missing items were scored zero ${ }^{15}$ and a maximum of five were allowed to be missing. The GHQ-30 focuses on inability to carry out normal functions and the appearance of new and distressing phenomena and due to the 'as usual' wording is sensitive to short term rather than chronic disorders. $^{2}$

\section{Baseline covariates}

Covariates were chosen based on related literature including reviews ${ }^{16-20}$ and individual studies ${ }^{7}{ }^{21-23}$ and on consideration of the likely mechanisms linking CMD to mortality. ${ }^{24} 25$ Potential confounders were sex, age (cohort), serious physical illness and social class; and potential mediators were health behaviours: smoking, alcohol consumption, pulse rate, obesity and living alone, which may also be confounders. Serious physical illness was measured as the reported number (none, one, two or more) of any of the following conditions which were classified using Royal College of General Practitioners $\operatorname{codes}^{26}$ : CVD (1940-1950, 2100-2115), hypertension (1910), diabetes (720), serious respiratory conditions $(2475,2490,2495,2505$, $2510,2515,2520,2525,2530)$ and any cancer (0400-0590). The conditions were obtained from the open-ended responses to a series of prompts on longstanding illness, disability or infirmity; other serious health problems; mental health problems and from a categorical list of common conditions. Social class was measured by occupation, coded according to the Registrar General's 1980 classification $^{27}$ for current or previous occupation of the respondent or their partner using the highest status class and dichotomised into manual and non-manual. Smokers were classified as never, ex or current smokers. Alcohol consumption was asked using a 7-day recall table and categorised into non-drinkers, safe drinkers and binge drinkers (consuming half the recommended weekly limit in 1 day: seven or more units per day for women, 10 or more for men). ${ }^{28}$ Using height and weight measured by the nurse, body mass index (BMI) was calculated and those with a BMI of 30 or more were classified as obese. Owing to the lack of consistent physical activity data across the two cohorts at baseline, pulse rate in beats per minute which was measured by the nurse was used as a proxy for physical activity/fitness. ${ }^{29}$ Living alone was a dichotomous variable based on the number of people in the household.

\section{STATISTICAL METHODS}

Baseline characteristics of participants, and Spearman rank correlations between GHQ-30 and HADS scores were obtained using SPSS V.19.

Using STATA V.11.0, Cox Proportional Hazards models were run with age at death, or age at June 2011 if still alive, as the time variable. Robust SEs were calculated and a dummy variable for locality included in all models to allow for the complex sample design. The proportional hazards assumption was checked using a test based on Schoenfeld residuals and was found to hold for all measures of CMD and for all covariates except cohort, so the Cox regression analyses were stratified by cohort using STATA's strata subcommand. Interactions with cohort, sex and with serious physical illness were considered plausible and investigated. Non-linear (quadratic) relationships between each measure of CMD and mortality were tested, but none were significant (so results not shown). Each measure of CMD was transformed into z-scores so that the HRs were on a similar scale and therefore more comparable across measures and the results presented as the HR per SD in score. Covariates were added to the model cumulatively in order to understand 
their effect on the CMD and mortality association: potential confounders were added before potential mediators: model (1) adjusted for cohort and sex; then serious illness was added: model (2); then social class was added: model (3); then smoking was added: model (4); and binge drinking, obesity, pulse and living alone were added for the fully adjusted model: model (5). To assess the impact of missing data among those with available CMD data, the basic relationships were compared for those with and without missing covariates.

\section{RESULTS}

Of the 2995 individuals from the 1930s and 1950s cohorts of the Twenty-07 Study, 2776 were screened at 'baseline' (ie, Wave 1 for the 1930s cohort and Wave 2 for the 1950s cohort), and of these the 2547 with complete data for all measures were included in the analysis (figure 1). Those with missing data were more likely to be in the 1930 s cohort, to have died, and to be in the manual social classes. A sensitivity analysis in those with CMD measures showed a similar association between CMD and mortality for those with and without missing covariates (results available on request). Table 1 shows the baseline characteristics of participants by vital status, and by GHQ-30, HADS-D and HADS-A scores: of the 2547 individuals, 687 (27\%) had died in the follow-up period. These were more likely to be men and to be in the 1930s cohort. The mean scores of the CMD measures are shown in table 1 . The Spearman rank correlations with GHQ-30 were: for HADS-D, $0.570(\mathrm{p}<0.001)$ and for HADS-A, $0.630(\mathrm{p}<0.001)$.

For all-cause mortality, the HRs per SD increase in CMD score are reported in table 2. After adjustment for sex and cohort, all the measures of CMD were significantly related to all-cause mortality, with similar HRs for HADS-D and GHQ-30, and the HR for the specific measure of anxiety, HADS-A, the smallest. Adjustment for the covariates attenuated the HRs, with most attenuation due to serious illness or smoking and with social class being the next most important. Serious illness attenuated the HRs most for HADS-D and GHQ-30, while smoking attenuated them most for HADS-A. After full adjustment the HRs for HADS-D and GHQ-30 were still similar and the smallest HR for HADS-A was no longer significant. The only significant interaction was between HADS-A and sex: in subgroup analyses for HADS-A the fully adjusted HR for men was 1.16 (95\% CI 1.02 to 1.32 ), comparable in magnitude with the HR for HADS-D, and for women was not significant (HR 0.94 (95\% CI 0.85 to 1.03)).

For cause-specific mortality, the number of deaths from each cause and the HRs per SD increase in scores are reported in table 3 with only Models (1) and (5) shown. After full

Table 1 Baseline characteristics of participants according to vital status and CMD scores

\begin{tabular}{|c|c|c|c|c|c|c|c|c|c|c|}
\hline \multirow[b]{2}{*}{ Baseline covariates } & \multicolumn{2}{|l|}{ All } & \multicolumn{2}{|c|}{ Died } & \multicolumn{2}{|c|}{ GHQ-30 } & \multicolumn{2}{|c|}{ HADS-A } & \multicolumn{2}{|c|}{ HADS-D } \\
\hline & $\mathrm{N}$ & $(\%)$ & $\mathrm{N}$ & $(\%)$ & Mean & (SD) & Mean & (SD) & Mean & (SD) \\
\hline All & 2547 & $(100)$ & 687 & (27.0) & 25.3 & $(10.8)$ & 7.6 & (3.9) & 4.3 & $(3.1)$ \\
\hline \multicolumn{11}{|l|}{ Sex } \\
\hline Men & 1143 & (44.9) & 368 & $(32.2)$ & 24.4 & $(10.7)$ & 6.9 & (3.8) & 4.2 & (3.1) \\
\hline Women & 1404 & $(55.1)$ & 319 & (22.7) & 26.0 & $(10.9)$ & 8.1 & $(4.0)$ & 4.4 & $(3.1)$ \\
\hline \multicolumn{11}{|l|}{ Cohort } \\
\hline 1950s & 1163 & $(45.7)$ & 83 & $(7.1)$ & 24.4 & $(10.9)$ & 7.4 & (3.8) & 3.9 & $(3.0)$ \\
\hline 1930 s & 1384 & $(54.3)$ & 604 & (43.6) & 26.0 & $(10.8)$ & 7.8 & $(4.0)$ & 4.7 & (3.2) \\
\hline \multicolumn{11}{|l|}{ Serious illness } \\
\hline None & 2094 & (82.2) & 451 & (21.5) & 24.5 & $(10.4)$ & 7.4 & (3.9) & 4.1 & (3.0) \\
\hline One & 380 & (14.9) & 186 & (48.9) & 27.8 & $(12.0)$ & 8.0 & $(4.0)$ & 5.1 & (3.4) \\
\hline Two or more & 73 & (2.9) & 50 & (68.5) & 32.8 & (12.6) & 9.4 & (3.8) & 6.6 & (3.3) \\
\hline \multicolumn{11}{|l|}{ Social class } \\
\hline Non-manual & 1564 & (61.4) & 310 & (19.8) & 24.0 & (9.6) & 7.2 & (3.7) & 3.9 & $(2.9)$ \\
\hline Manual & 983 & (38.6) & 377 & (38.4) & 27.3 & (12.3) & 8.1 & $(4.2)$ & 5.0 & (3.4) \\
\hline \multicolumn{11}{|l|}{ Smoker } \\
\hline Never & 923 & (36.2) & 141 & (15.3) & 23.8 & $(9.8)$ & 3.9 & (2.9) & 7.1 & (3.7) \\
\hline Ex & 535 & (21.0) & 132 & (24.7) & 24.6 & (10.4) & 4.1 & (3.0) & 7.2 & (3.9) \\
\hline Current & 1089 & (42.8) & 414 & (38.0) & 26.9 & (11.7) & 4.8 & (3.3) & 8.1 & $(4.1)$ \\
\hline \multicolumn{11}{|l|}{ Binge drinking } \\
\hline Non-drinkers & 788 & (30.9) & 229 & (29.1) & 26.8 & $(12.2)$ & 4.8 & (3.4) & 7.9 & $(4.2)$ \\
\hline Not binge drinkers & 1325 & (52.0) & 314 & (23.7) & 24.2 & (9.7) & 4.0 & (2.9) & 7.3 & (3.7) \\
\hline Binge drinkers & 434 & (17.0) & 144 & (33.2) & 25.8 & (11.0) & 4.4 & (3.2) & 7.6 & $(4.0)$ \\
\hline \multicolumn{11}{|l|}{ Obese $(\mathrm{BMI} \geq 30)$} \\
\hline No & 2163 & (84.9) & 567 & (26.2) & 25.2 & (10.9) & 4.2 & (3.2) & 7.6 & (3.9) \\
\hline Yes & 384 & (15.1) & 120 & (31.3) & 25.6 & (10.6) & 4.7 & (3.0) & 7.4 & (3.9) \\
\hline \multicolumn{11}{|l|}{ Lives alone } \\
\hline No & 2286 & (89.8) & 585 & (25.6) & 24.9 & (10.6) & 4.3 & (3.1) & 7.5 & (3.9) \\
\hline Yes & 261 & (10.2) & 102 & (39.1) & 28.3 & (12.1) & 4.8 & (3.5) & 8.5 & (4.3) \\
\hline \multicolumn{11}{|l|}{ Pulse mean (SD) } \\
\hline All & 71.0 & (9.8) & \multicolumn{2}{|c|}{ Correlation: } & \multicolumn{2}{|c|}{0.087} & \multicolumn{2}{|c|}{0.079} & \multicolumn{2}{|c|}{0.050} \\
\hline Alive & 70.0 & (9.5) & & & & & & & & \\
\hline Died & 73.8 & $(10.0)$ & & & & & & & & \\
\hline
\end{tabular}

BMI, body mass index; CMD, common mental disorder; GHQ-30, 30-item General Health Questionnaire; HADS, Hospital Anxiety and Depression Scale. 
Table 2 HR per SD increase (95\% Cls) for the association of GHQ and HADS scores with all-cause mortality

\begin{tabular}{llll}
\hline HR per SD increase (95\% CIs) & & & HADS-A \\
All-cause (687 deaths) & GHQ-30 & $1.15(1.06$ to 1.26$)$ & HADS-D \\
Model (1): adj sex, cohort & $1.27(1.16$ to 1.39$)$ & $1.12(1.02$ to 1.22$)$ & $1.30(1.21$ to 1.41$)$ \\
Model (2): adj (1)+serious illness & $1.21(1.11$ to 1.33$)$ & $1.08(0.99$ to 1.18$)$ & $1.24(1.14$ to 1.35$)$ \\
Model (3): adj (2)+social class & $1.17(1.07$ to 1.28$)$ & $1.04(0.95$ to 1.14$)$ & $1.20(1.10$ to 1.30$)$ \\
Model (4): adj (3)+smoking & $1.13(1.04$ to 1.24$)$ & $1.05(0.96$ to 1.14$)$ & $1.16(1.07$ to 1.27$)$ \\
Model (5): fully adjusted* & $1.13(1.04$ to 1.23$)$ & \\
\hline${ }^{*}$ Adjusted for sex, cohort, serious illness, social class, smoking, alcohol, obesity, pulse and living alone. & & $1.15(1.07$ to 1.25$)$ \\
GHQ-30, 30-item General Health Questionnaire; HADS, Hospital Anxiety and Depression Scale.
\end{tabular}

adjustment there were significant HRs for CVD mortality and all CMD measures with HRs for HADS-D and GHQ-30 being largest, and the smallest HR was for HADS-A. For respiratory mortality there was a significant association after full adjustment with GHQ-30 but with neither of the HADS measures. For cancer mortality there was no significant association with any of the CMD measures with all HRs around one. Although numbers are small, results are reported for mortality from injuries (including two suicides) HRs (all non-significant) were the highest for HADS-D, then HADS-A and very low HRs for GHQ-30. For mortality from 'other' causes, after full adjustment, there was a significant association with HADS-D only.

\section{DISCUSSION}

In the present analyses, comprising two cohorts, we found that various measures of anxiety and depression were related to an elevated risk of total and cause-specific mortalities. HADS-D and GHQ-30 were similar in their relationships to all-cause, CVD, and lack of relationship to cancer mortality. There were some cause-specific differences in that after full adjustment the only significant HR for respiratory mortality was for GHQ-30 and for mortality from 'other' causes was for HADS-D. HRs for HADS-A tended to be lower than that for the other measures. For both HADS-D and GHQ-30, existing physical illness was most important in attenuating the association with all-cause mortality, followed by smoking.

Although a number of studies have found an association between CMD and all-cause mortality, ${ }^{3-5} 9$ 30-32 to our knowledge no others have compared GHQ-30 and the HADS subscales in this respect. Some studies have compared measures of anxiety and depression and found as we do, that depression is a stronger predictor than anxiety ${ }^{31}$ while others found the opposite. ${ }^{9}$ The HUNT study which used the HADS scale ${ }^{5}$ found, like us, a strong association with HADS-D and all-cause mortality, but a non-linear association for HADS-A, which we did not find. We do not believe this difference is due to our much longer follow-up since the proportional hazards assumption held; however the HUNT study included younger participants and they did find an interaction with age in some of their analyses, so the difference observed may be due to the different ages in the two studies. The HUNT study also found an association with anxiety only (ie, without comorbid depression), adjusted for age, was significant in men but not in women which is consistent with our interaction between HADS-A and sex.

In relation to cause-specific mortality, a review ${ }^{17}$ shows that many studies find that CMD predicts CVD morbidity, but there are fewer studies of CMD predicting CVD mortality, ${ }^{3} 493032$ and even fewer including more than one measure of CMD. ${ }^{6} 9$ The US military veterans study showed that depression predicted CVD mortality less strongly than anxiety, ${ }^{9}$ while another study showed that HADS depression scores predicted CVD mortality while HADS anxiety scores were protective. ${ }^{6}$ The analysis presented here showed that after full adjustment, HADS-D and GHQ-30 had similar-sized HRs for CVD mortality and that those for anxiety were smaller. Respiratory mortality was found to be significantly related to GHQ-30 and more weakly to HADS-D in our analysis but this outcome has been included in only a small number of studies. ${ }^{4633}$ Consistent with this, one study found that HADS-D score but not HADS-A score was related to respiratory mortality ${ }^{6}$ and the OR was higher than that for CVD mortality. Another study using the Present State Examination $^{33}$ found that depression predicted respiratory mortality in women but this study did not adjust for any potential confounders such as smoking. An analysis using UK Health and Lifestyle Survey found that although GHQ-30 predicted respiratory mortality, this was explained by confounders and mediators. ${ }^{4}$ The lack of association between CMD and cancer mortality is consistent with null or weak findings of other studies $^{3} 4$ 33-36 although one study did find a significant association between HADS-D alone (ie, without comorbid anxiety) and cancer mortality. ${ }^{6}$ Relationships between mental illness and cancer tend to be partially explained by pre-existing cancer, ${ }^{35} 37$ suggesting the relationships observed were partly due to the psychological effect of the cancer diagnosis. Even before adjusting for existing illness, we found very weak relationships between CMD and cancer mortality, and with a very small number with existing cancer (only 35 people), this may be why we found such weak relationships. There were various strengths in this study: more than one measure of CMD which is rarely available; a variety of covariates were measured, including those recommended in the literature ${ }^{16}$ : serious illness, smoking, alcohol; the data were from a general rather than clinical population and prevalence rates of anxiety and depression have been shown to be similar to normative studies $^{38}$; a long period of follow-up; and there were data for both men and women. There were also some limitations: $8 \%$ of the original sample had missing data and were excluded; however, a sensitivity analysis showed no bias in the association between CMD and mortality among those with missing covariates but available CMD data. Although a good range of appropriate covariates were included, it is possible that there is some omitted variable bias or measurement error. For example, pulse had to be used in lieu of physical activity, although this has been shown to be a good proxy. ${ }^{29}$ There appears to be low power for causes other than CVD and cancer due to fewer deaths. It is important to note that neither HADS nor GHQ-30 scores represent clinical diagnoses of mental disorders but are screening instruments.

The similarities between GHQ-30 and HADS-D in relation to CVD mortality may be explained in both measures being designed to measure symptoms typical of depression. Both GHQ-30 and HADS were designed to be unaffected by physical illness: adjusting for existing physical illness similarly attenuated 
Table 3 HR per SD increase ( $95 \%$ Cls) for the association of GHQ and HADS scores with cause-specific mortality

\begin{tabular}{|c|c|c|c|}
\hline \multicolumn{4}{|l|}{ HR per SD increase $(95 \% \mathrm{Cls})$} \\
\hline & GHQ-30 & HADS-A & HADS-D \\
\hline \multicolumn{4}{|l|}{ CVD (254 deaths) } \\
\hline Model (1): adj sex, cohort & 1.44 (1.27 to 1.62$)$ & 1.30 (1.13 to 1.49$)$ & $1.46(1.27$ to 1.67$)$ \\
\hline Model (5): fully adjusted* & $1.24(1.11$ to 1.40$)$ & 1.15 (1.01 to 1.32$)$ & $1.24(1.07$ to 1.43$)$ \\
\hline \multicolumn{4}{|l|}{ Respiratory (76 deaths) } \\
\hline Model (1): adj sex, cohort & $1.52(1.30$ to 1.79$)$ & 1.27 (1.02 to 1.56$)$ & $1.42(1.17$ to 1.73$)$ \\
\hline Model (5): fully adjusted* & 1.33 (1.13 to 1.55$)$ & 1.09 (0.89 to 1.33$)$ & $1.21(0.99$ to 1.48$)$ \\
\hline \multicolumn{4}{|l|}{ Cancer (250 deaths) } \\
\hline Model (1): adj sex, cohort & 1.04 (0.90 to 1.21$)$ & 1.00 (0.85 to 1.16$)$ & $1.10(0.97$ to 1.26$)$ \\
\hline Model (5): fully adjusted a & 0.95 (0.81 to 1.11$)$ & $0.92(0.79$ to 1.08$)$ & $1.00(0.88$ to 1.15$)$ \\
\hline \multicolumn{4}{|l|}{ Injuries (17 deaths) } \\
\hline Model (1): adj sex, cohort & $0.78(0.43$ to 1.41$)$ & $1.17(0.73$ to 1.90$)$ & 1.35 (0.81 to 2.24$)$ \\
\hline Model (5): fully adjusted* & $0.73(0.43$ to 1.23$)$ & 1.15 (0.70 to 1.86$)$ & 1.35 (0.80 to 2.28$)$ \\
\hline \multicolumn{4}{|l|}{ Other (90 deaths) } \\
\hline Model (1): adj sex, cohort & 1.29 (1.03 to 1.62$)$ & $1.14(0.92$ to 1.42$)$ & $1.37(1.14$ to 1.64$)$ \\
\hline Model (5): fully adjusted* & 1.21 (0.93 to 1.57$)$ & 1.07 (0.84 to 1.35$)$ & $1.28(1.05$ to 1.55$)$ \\
\hline
\end{tabular}

the HRs for GHQ-30 and HADS-D. There was also consistency across measures in the finding of no relationship with cancer mortality indicating no psychological link in this sample which has a small number of cases of diagnosed cancer at baseline. Although there were differences in significance between GHQ-30 and HADS-D for respiratory and 'other' mortality, the HRs were not hugely different between the measures and low power may be an explanation.

Various biological mechanisms linking CMD to CHD in particular have been proposed ${ }^{24} 25$ including hypothalamicpituitary-adrenocortical (HPA) reactivity, diminished heart rate variability, myocardial ischaemia, ventricular instability in reaction to mental stress and alterations in platelet receptors. These could explain the remaining association between all the measures of CMD and CVD mortality after full adjustment. In relation to respiratory mortality, it is possible that as well as influences on the circulatory system, ${ }^{24}$ some of the same mechanisms could increase the risk of infections in the lungs ${ }^{39}$ or hypoxia, lack of oxygen, could affect mental health. ${ }^{40}$ It is important to note that although the measures of CMD were related to mortality, it cannot be concluded from this analysis that CMD causes mortality.

The similarities in the HRs for GHQ-30 and HADS-D in relation to all-cause and cause-specific mortality add strength to the existing evidence of a relationship between mental health and mortality, especially since recommended confounders and mediators were included in the analyses.

Future work in this area, including various causes of mortality as outcomes, would be valuable, especially in larger studies.

\section{What is already known on this subject?}

- Many studies, including some using the General Health Questionnaire (GHQ) or the Hospital Anxiety and Depression Scale (HADS), show that people with common mental disorders (CMD) have an increased risk of mortality.

- Differences have been found in how measures of depression and anxiety predict mortality but results are not consistent across studies and GHQ and HADS have not been compared in this respect.

\section{What this study adds?}

- There are remarkable similarities between GHQ-30 and HADS depression in their association with all-cause and cause-specific mortality.

- Various causes of mortality should be included as outcomes in studies of CMD and mortality, especially in larger studies.

Acknowledgements We are grateful to all of the participants in the study, and to the survey staff and research nurses who carried it out.

Contributors MB and GDB had the idea for the analysis. MKH performed the analyses and interpretation of results. MB and GDB discussed results with MKH and advised on the direction of the work. MKH did the literature review and drafted the manuscript. MB and GDB critically commented on the scientific content of the manuscript and approved the final draft.

Funding The West of Scotland Twenty-07 Study is funded by the UK Medical Research Council (MRC) (MC_A540_53462) and the data were originally collected by the MRC Social and Public Health Sciences Unit. MK Hannah and M Benzeval are funded by the MRC (MC A540_5TK10) and GD Batty is a Wellcome Trust Fellow.

Competing interests None.

Ethics approval The ethics subcommittee of the West of Scotland Area Medical Committees and the GP Sub-Committee of Greater Glasgow Health Board.

Provenance and peer review Not commissioned; externally peer reviewed.

Data sharing statement Applications from bona fide researchers to use existing Twenty-07 data are welcome. All potential collaborators work with a link person, one of the Twenty-07 coinvestigators, to support their application and data analysis. Our Study website has further information:- http://2007study.sphsu.mrc.ac.uk/.

Open Access This is an Open Access article distributed in accordance with the Creative Commons Attribution Non Commercial (CC BY-NC 3.0) license, which permits others to distribute, remix, adapt, build upon this work non-commercially, and license their derivative works on different terms, provided the original work is properly cited and the use is non-commercial. See: http://creativecommons.org/ licenses/by-nc/3.0/

\section{REFERENCES}

1 Snaith RP, Zigmond AS. The Hospital Anxiety and Depression Scale with the Irritability-Depression-Anxiety Scale and the Leeds Situational Anxiety Scale manual. Windsor: NFER-Nelson, 1994.

2 Goldberg D, Williams P. A user's guide to the General Health Questionnaire. Windsor: NFER-Nelson, 1988. 
3 Russ TC, Stamatakis E, Hamer M, et al. Association between psychological distress and mortality: individual participant pooled analysis of 10 prospective cohort studies. BMJ 2012;345:e4933.

4 Batty GD, Hamer M, Der G. Does somatic illness explain the association between common mental disorder and elevated mortality? Findings from extended follow-up of study members in the UK Health and Lifestyle Survey. J Epidemiol Community Health 2012;66:647-9.

5 Mykletun A, Bjerkeset 0 , Overland S, et al. Levels of anxiety and depression as predictors of mortality: the HUNT study. Br J Psychiatry 2009;195:118-25.

6 Mykletun A, Bjerkeset 0, Dewey M, et al. Anxiety, depression, and cause-specific mortality: the HUNT study. Psychosom Med 2007;69:323-31.

7 Rasul F, Stansfeld SA, Smith G Davey, et al. Psychological distress, physical illness and risk of myocardial infarction in the Caerphilly study. Psychol Med 2007:37:1305-13.

8 Rasul F, Stansfeld SA, Hart CL, et al. Psychological distress, physical illness, and risk of coronary heart disease. J Epidemiol Community Health 2005;59:140-5.

9 Phillips AC, Batty GD, Gale CR, et al. Generalized anxiety disorder, major depressive disorder, and their comorbidity as predictors of all-cause and cardiovascular mortality: the Vietnam experience study. Psychosom Med 2009;71:395-403.

10 O'Rourke S, MacHale S, Signorini D, et al. Detecting psychiatric morbidity after stroke: comparison of the GHQ and the HAD Scale. Stroke 1998:29:980-5.

11 Benzeval M, Der G, Ellaway A, et al. Cohort profile: West of Scotland Twenty-07 Study - health in the community. Int J Epidemiol 2009;38:1215-23.

12 Der G. A comparison of the West of Scotland Twenty-07 Study sample and the 1991 census SARs. MRC Medical Sociology Unit Working Paper. Glasgow: MRC Medical Sociology Unit, 1998.

13 Bjelland I, Dahl AA, Haug TT, et al. The validity of the Hospital Anxiety and Depression Scale: an updated literature review. J Psychosom Res 2002;52:69-77.

14 GL Assessment. Hospital Anxiety and Depression Scale, Frequently Asked Questions (FAQs). Secondary Hospital Anxiety and Depression Scale, Frequently Asked Questions (FAQs). 2013. http://www.gl-assessment.co.uk/products/hospital-anxiety-anddepression-scale/hospital-anxiety-and-depression-scale-faqs (accessed 13 Feb 2013).

15 GL Assessment. General Health Questionnaire, Frequently Asked Questions (FAQs) Secondary General Health Questionnaire, Frequently Asked Questions (FAQs). 2013. http://www.gl-assessment.co.uk/products/general-health-questionnaire/faqs (accessed 13 Feb 2013).

16 Wulsin L, Vaillant G, Wells V. A systematic review of the mortality of depression. Psychosom Med 1999;61:6-17.

17 Suls J, Bunde J. Anger, anxiety, and depression as risk factors for cardiovascular disease: the problems and implications of overlapping affective dispositions. Psychol Bull 2005;131:260-300.

18 Chida Y, Steptoe A. Positive psychological well-being and mortality: a quantitative review of prospective observational studies. Psychosom Med 2008:70:741-56

19 Rugulies R. Depression as a predictor for coronary heart disease: a review and meta-analysis. Am J Prev Med 2002;23:51-61.

20 Hemingway $\mathrm{H}$, Marmot M. Psychosocial factors in the aetiology and prognosis of coronary heart disease: systematic review of prospective cohort studies. BMJ 1999;318:1460-7.

21 Hamer M, Molloy GJ, Stamatakis E. Psychological distress as a risk factor for cardiovascular events: pathophysiological and behavioral mechanisms. J Am Coll Cardiol 2008;52:2156-62
22 Kauhanen J, Kaplan GA, Goldberg DE, et al. Beer binging and mortality: results from the Kuopio ischaemic heart disease risk factor study, a prospective population based study. BMJ 1997;315:846-51.

23 Henderson M, Hotopf M, Shah I, et al. Psychiatric disorder in early adulthood and risk of premature mortality in the 1946 British Birth Cohort. BMC Psychiatry 2011;11:37.

24 Musselman DL, Evans DL, Nemeroff CB. The relationship of depression to cardiovascular disease: epidemiology, biology, and treatment. Arch Gen Psychiatry 1998:55:580-92.

25 Carney R, Freedland K, Miller G, et al. Depression as a risk factor for cardiac mortality and morbidity: a review of potential mechanisms. J Psychosom Res 2002;53:897-902.

26 The Royal College of General Practitioners. Occasional Paper No 26. The classification and analysis of general practice data. 2nd edn. London: The Royal College of General Practitioners, 1986.

27 Office of Population Censuses and Surveys. Classification of occupations. London: HMSO, 1980.

28 Moore L, Smith C, Catford J. Binge drinking: prevalence, patterns and policy. Health Educ Res 1994:9:497-505.

29 Blair SN, Kannel WB, Kohl HW, et al. Surrogate measures of physical activity and physical fitness. Evidence for sedentary traits of resting tachycardia, obesity, and low vital capacity. Am J Epidemiol 1989;129:1145-56.

30 Gump BB, Matthews KA, Eberly LE, et al. Depressive symptoms and mortality in men: results from the Multiple Risk Factor Intervention Trial. Stroke 2005:36:98-102.

31 Wilson RS, Bienias JL, Mendes de Leon CF, et al. Negative affect and mortality in older persons. Am J Epidemiol 2003;158:827-35.

32 Marzari C, Maggi S, Manzato E, et al. Depressive symptoms and development of coronary heart disease events: the Italian longitudinal study on aging. J Gerontol $A$ Biol Sci Med Sci 2005:60A(1):85-92.

33 Joukamaa $M$, Heliovaara $M$, Knekt $P$, et al. Mental disorders and cause-specific mortality. Br J Psychiatry 2001:179:498-502.

34 Oerlemans $M$, van den Akker M, Schuurman A, et al. A meta-analysis on depression and subsequent cancer risk. Clin Pract Epidemiol Ment Health 2007:3:29.

35 Hamer M, Chida Y, Molloy GJ. Psychological distress and cancer mortality. J Psychosom Res 2009:66:255-8.

36 Osborn DJ, Levy G, Nazareth I, et al. Relative risk of cardiovascular and cancer mortality in people with severe mental illness from the United Kingdom's general practice research database. Arch Gen Psychiatry 2007;64:242-9.

37 Whitley E, Batty GD, Mulheran PA, et al. Psychiatric disorder as a risk factor for cancer: different analytic strategies produce different findings. Epidemiology 2012;23:543-50

38 Green MJ, Benzeval M. Ageing, social class, and common mental disorders: longitudinal evidence from three cohorts in the West of Scotland. Psychol Med 2011:41:565-74.

39 Pembroke TPI, Rasul F, Hart CL, et al. Psychological distress and chronic obstructive pulmonary disease in the Renfrew and Paisley (MIDSPAN) study. J Epidemiol Community Health 2006;60:789-92.

40 Mikkelsen RL, Middelboe T, Pisinger C, et al. Anxiety and depression in patients with chronic obstructive pulmonary disease (COPD). A review. Nord J Psychiatry 2004;58:65-70 